\title{
Between Scylla and Charybdis: Difficult Balance between Anticoagulation and Bleeding in the Management of LVAD Thrombosis: A Case Report
}

Perna E*, Cipriani MG*, De Marco F, Macera F, Milazzo F, Garascia A, Ammirati E, D'Angelo L, Gagliardone MP, Russo CF and Frigerio M

De Gasperis Cardio Center, Niguarda Ca' Granda Hospital, Italy

\begin{abstract}
Case: The patient was a 67 years old male. The patient experienced an episode of LVAD thrombosis. Device replacement was not possible due to high surgical risk. Pharmacological strategy was adopted with intraventricular fibrinolysis+intravenous Tirofiban infusion.

Outcome: Despite thrombosis resolution, two episodes of bleeding occurred. Thus we lowered antithrombotic drugs dose with subsequent relapse risk. At the end, a successful therapeutic strategy was achieved.

Conclusion: Our case report shows how the balance between anticoagulation and bleeding is a tricky feature of LVAD thrombosis management. When device replacement is not an option, trying to remove the clot soon with aggressive antithrombotic therapy can be a winning strategy and can quickly stop the haemolysis. Unfortunately, it can produce major bleeding events, harmful and potentially life-threatening. To be aware of potential cause of bleeding and looking for the appropriate dosage of antithrombotic drugs can be useful to manage these complications
\end{abstract}

Keywords: Ventricular assist device; Thrombus; Pump thrombosis; Haemolysis; Lytic therapy; GpIIb/IIIa inhibitor; Anti-coagulation; Heart ware

\section{Abbreviations}

APTT: Activated Partial Thromboplastin Time; ASA: Acetylsalicylic Acid; CT: Computed Tomography; FDP: Fibrinogen Degradation Product; Hb: Haemoglobin; HF: Heart Failure; HVAD: Heart Ware Ventricular Assistance Device; INR: International Normalised Ratio; IV: Intravenous; LDH: Lactate Dehydrogenase; LVAD: Left Ventricular Assistance Device; MA: Maximal Amplitude; OAC: Oral Anticoagulant; pfHg: Plasma-Free Haemoglobin; PLTs: Platelets; TEG: Thromboelastography

\section{Introduction}

Continuous flow Left Ventricular Assist Devices (LVAD) is used in patients with advanced heart failure. Device-related hematologic complications remain an important associated morbidity. Pump thrombosis is a major and life-threatening adverse event. Despite chronic anticoagulation with warfarin, the Heart Ware Investigators noted high incidence (0.063-0.08 events/patient-year) of thrombosis in the Heart Ware HVAD Ventricular Assist System [1]. In this setting, the optimal treatment strategy is still uncertain. Treatment approaches include optimization of anticoagulation or antiplatelet therapy, fibrinolysis and device-exchange. However, when a surgical LVAD exchange is associated with a high morbidity and mortality, medical therapy can be an alternative strategy.

We report a case of pump thrombosis treated with medical therapy.

\section{Case Presentation}

The patient was a 67-years-old man, $170 \mathrm{~cm} \times 72 \mathrm{Kg}(\mathrm{BMI}$ 24.9), with a long history of ischemic cardiopathy, type II diabetes mellitus and atherosclerotic disease of carotid arteries. On March 2013 he underwent Heart Ware HVAD implantation as destination therapy, through sternotomy. Surgery and postoperative progress were uneventful and the patient was home discharge. Standard antithrombotic therapy was administered (warfarin with INR range 2-3, ASA $100 \mathrm{mg} /$ die, Dipyridamole $800 \mathrm{mg} / \mathrm{die}$ ). During the following months he remained in satisfactory clinical condition and HVAD parameters were within normal limits. On May 2014, haematuria occurred and he was readmitted to our hospital. No clinical signs of pulmonary or abdominal congestion or signs of cardiogenic shock were observed during first physical examination. HVAD parameters showed a progressive increase of Power (P $2.8 \rightarrow 3.6 \mathrm{~W}$ ) and calculated flow (F $3.9 \rightarrow 6 \mathrm{~L} / \mathrm{min}$ ), with a Speed (S) of $2360 \mathrm{rpm}$. Laboratory tests showed a significant elevation of serum lactate dehydrogenase (LDH) $>1547$ $\mathrm{U} / \mathrm{L}$ and plasma-free haemoglobin $(\mathrm{pfHg})>1046 \mathrm{mg} / \mathrm{L}$, a decrease in haptoglobin and anemia ( $\mathrm{Hb} 12.7 \mathrm{~g} / \mathrm{dL}$ ): major haemolysis was occurring. The anticoagulation profile was in range (INR 2.6, APTT $44 \mathrm{sec}$ ). Transesophageal echocardiography was performed: no clot was found at the inflow and outflow cannula site, but there was left ventricle dilatation with frequent aortic valve opening.

\section{A diagnosis of suspect HVAD thrombosis was made.}

Our patient had a high surgical risk, so since the beginning we ruled out device replacement. A continuous infusion of intravenous heparin (target activated partial thromboplastin time of 50-60 seconds) was started, adding greater aspirin dose, $300 \mathrm{mg} /$ die. Nevertheless, HVAD parameters continued to worsen $(\mathrm{F}>10)$ and after 24 hours patient became "cold and wet" (peripheral hypoperfusion, central venous pressure $>19 \mathrm{~mm} \mathrm{Hg}$ ). Therefore, the decision to perform fibrinolysis

*Corresponding authors: Perna E, De Gasperis Cardio Center, Niguarda Ca' Granda Hospital, Piazza Ospedale Maggiore 3, 20162 Milan, Italy, Tel: +393332106609; Fax: 02 64442566; E-mail: enrico.perna@ospedaleniguarda.it

Cipriani MG, De Gasperis Cardio Center, Niguarda Ca' Granda Hospital, Piazza Ospedale Maggiore 3, 20162 Milan, Italy, Tel: +393332106609; Fax: 0264442566 ; E-mail: manlio.cipriani@ospedaleniguarda.it

Received January 04, 2016; Accepted February 10, 2016; Published February 15,2016

Citation: Perna E, Cipriani MG, Marco DF, Macera F, Milazzo F, et al. (2016) Between Scylla and Charybdis: Difficult Balance between Anticoagulation and Bleeding in the Management of LVAD Thrombosis: A Case Report. Cardiovasc Pharm Open Access 5: 173. doi:10.4172/2329-6607.1000173

Copyright: (C) 2016 Perna E, et al. This is an open-access article distributed under the terms of the Creative Commons Attribution License, which permits unrestricted use, distribution, and reproduction in any medium, provided the original author and source are credited. 
Citation: Perna E, Cipriani MG, Marco DF, Macera F, Milazzo F, et al. (2016) Between Scylla and Charybdis: Difficult Balance between Anticoagulation and Bleeding in the Management of LVAD Thrombosis: A Case Report. Cardiovasc Pharm Open Access 5: 173. doi:10.4172/23296607.1000173

Page 2 of 4

was made: $\mathrm{x}$-ray guided intra-ventricular thrombolysis was performed and Alteplase was administered (10 mg+10 mg+10 mg) (Figure 1). The injections were made directly into the device's inflow cannula through a $6 \mathrm{Fr}$ AL 2 catheter inserted through the radial artery. Every injection lasted 10 minutes and the interval between every bolus was $15-20$ minutes. Blood tests were quite stable: PLTS $123 \times 10^{-3} /$ microliter, INR 3.09, APTT $111.9 \mathrm{sec}$ (six hours later $50.4 \mathrm{sec}$ ), D-dimer 21.78 $\mathrm{mcg} / \mathrm{mL}$. After an initial normalization, HVAD parameters showed a new slight increasing trend without worsening of clinical conditions (hemodynamically stable patient). Intravenous antithrombotic therapy with glycoprotein IIb/IIIa inhibitors (Tirofiban) was started. The infusion of Tirofiban was conducted at a rate of $0.1 \mathrm{mcg} / \mathrm{Kg} / \mathrm{min}$, without bolus. Within 12 hours since the beginning, we observed first results and complete resolution was evident at day 4 with stable flows and power consumption (Figures 2 and 3 ).

In the next 20 days patient was fine and we tried to optimize his therapy, but a severe major bleeding event occurred, and patient

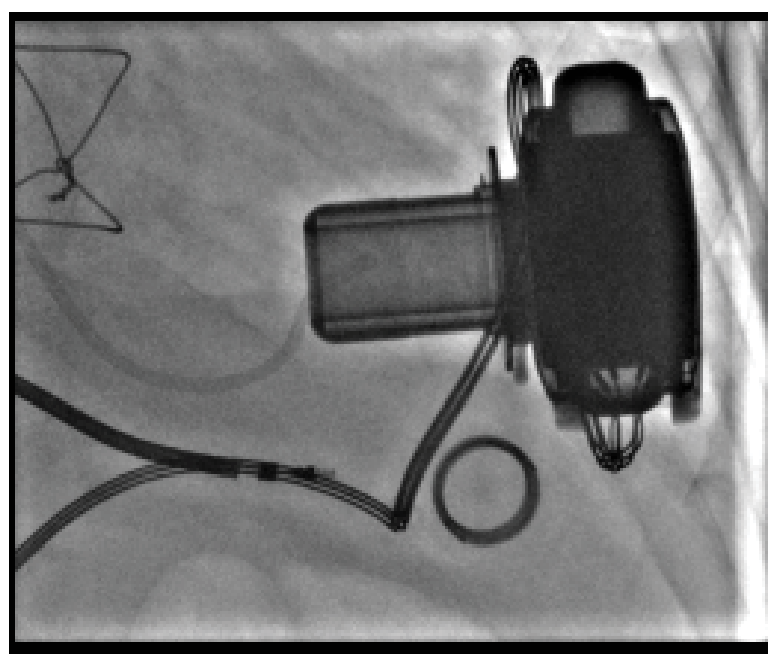

Figure 1: Intraventricular infusion of thrombolytic therapy: $x$-ray guided intraventricular thrombolysis with Alteplase. The injections were made directly into the device's inflow cannula through a 6Fr AL 2 catheter inserted through the radial artery. experienced massive bleeding from his urinary tract (bladder and prostatic tract of urethra). Endoscopic procedure and inferior bladder artery embolization were ineffective. The bleeding solved just after 25 days, multiple transfusions and frequent changes in dosage of antithrombotic therapy were performed (Aspirin $300 \mathrm{mg} \rightarrow 100$ $\mathrm{mg}$ and Intravenous Heparin APTT range $<50$ seconds). Five days after the resolution of haematuria, back pain with left lower limb irradiation showed up, a CT scan revealed a massive hematoma of the left psoas muscle, minimally stocked. The decision was made to stop heparin infusion and start OAC (warfarin) with INR range 2-2.5. No interventional procedures were necessary, bleeding stopped spontaneously.

After one week, HVAD parameters increased again. The patient was hemodynamically stable and a second infusion of Tirofiban $0.1 \mathrm{mg} /$ $\mathrm{Kg} / \mathrm{min}$ was started. After 48 hours parameters came back to normal and infusion was stopped; double antiplatelet therapy (Clopidogrel $75 \mathrm{mg}+$ ASA $100 \mathrm{mg}$ ) was started. On July $22^{\text {nd }}$ facing with haematuria relapse, clopidogrel was stopped and the following therapeutic regimen was set: ASA $100 \mathrm{mg}+$ Warfarin INR 2-2.2+low-molecular-weight heparin $4000 \mathrm{UI} /$ die.

Anticoagulation levels were reassessed twice a week according to thromboelastography. The last check (on the discharge day) showed stable anticoagulation status (native TPI 8,1) without platelets hyperaggregation (MA 69 in heparinase test). There were no more episodes of haematuria.

On August $8^{\text {th }}$ the patient was home discharged with the following blood tests: PLTs $228 \times 10^{-3} /$ microliter, Hb $10 \mathrm{~g} / \mathrm{dL}$, HcT $32.5 \%$, INR 2.56, D-dimer $1.63 \mathrm{mcg} / \mathrm{mL}$, FDP $482 \mathrm{mg} / \mathrm{dL}$.

\section{Discussion}

In the last years the number of advanced HF patients supported with LVADs is increased. Device-related hematologic complications remain an important cause of associated morbidity. Bleeding continue to be one of the most common adverse event reported [2]. Although rates of pump thrombosis and thromboembolic complications occur less frequently than before, they are often life-threatening associated with increased risk of both stroke and mortality [3]. Therefore, it is well recognised that device thrombosis is a clinical syndrome with

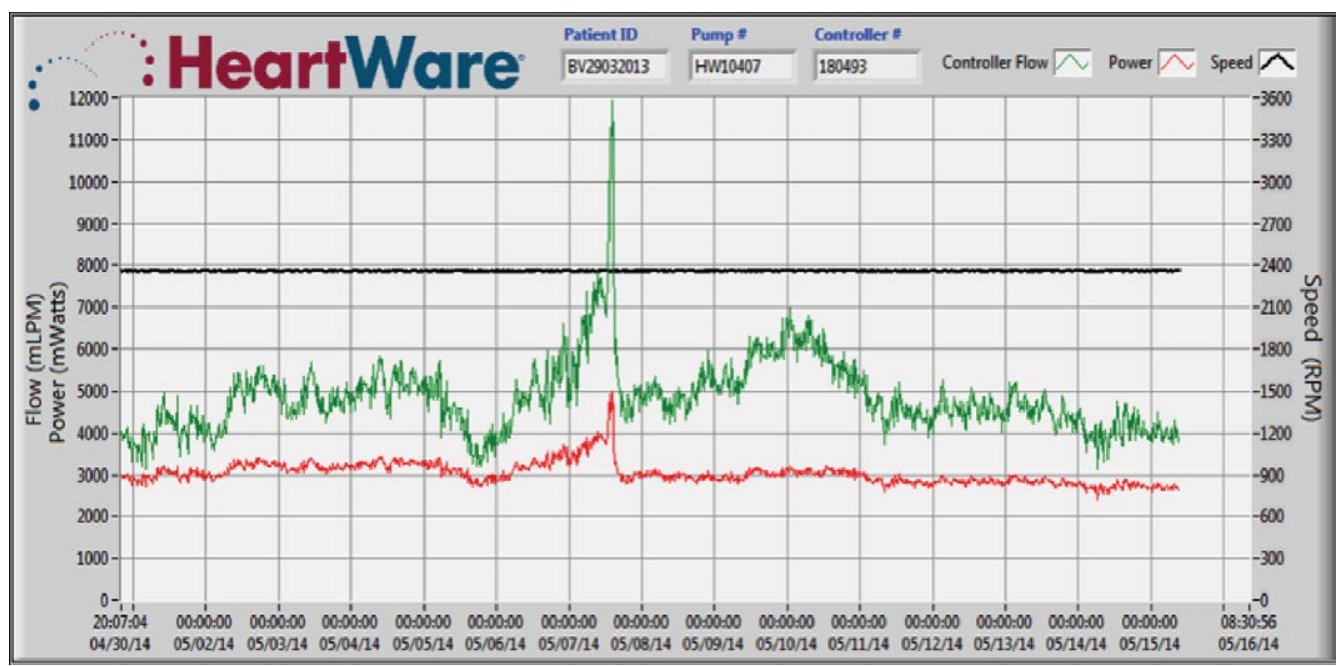

Figure 2: HVAD Parameters Log File: the high growth of the Flow value (red line) and of the Power (green line) and their reduction after fibrinolysis and anti-thrombotic therapy. 


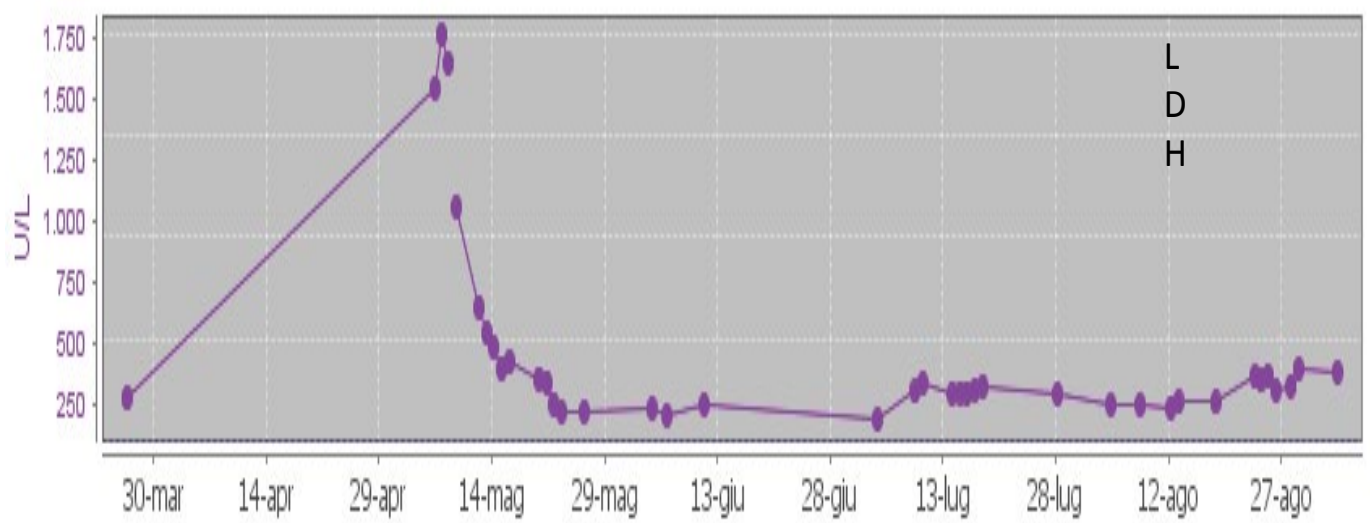

Figure 3: Lactate dehydrogenase's trend during the hospitalization with the sharp drop after fibrinolysis infusion: LDH is emerging as the most sensitive serial marker of thrombosis, with suggestions that an LDH value greater than $600 \mathrm{IU} / \mathrm{L}$ should prompt further evaluation.

several clinical, laboratory, echocardiographic and hemodynamic features. The most common clinical presentation of this adverse event is laboratory evidence of haemolysis [4-6]. The INTERMACS registry recently (2014) recategorized haemolysis in minor and major. Minor haemolysis is defined by the LDH or pfHb elevation in the absence of clinical signs/symptoms, and the management consists of anticoagulation optimization, increased aspirin doses or addition of a second antiplatelet therapy. Major haemolysis requires clinical symptoms or abnormal pump function to be diagnosed; hospital admission is recommended, as well as IV therapy with heparin and/or others additional treatment such as glycoprotein IIb/IIIa inhibitors or fibrinolytics especially if surgical intervention is contraindicated [6-7].

Our clinical case represents as a case of major haemolysis.

The decision to treat the patient just pharmacologically was driven by his clinical condition (fragility, organ damage) and his high surgical risk. The first step was antithrombotic therapy optimization with IV heparin infusion and increased aspirin dose. Careful monitoring of anticoagulation was performed with thromboelastography. Due to the rapid clinical deterioration with signs of shock, we decided to perform fibrinolysis. The benefit lasted 48 hours, and then the device parameters increased again, this time without clinical symptoms. To manage this second event, we decided to perform glycoprotein IIb/IIIa inhibitor (Tirofiban $0.1 \mathrm{gamma} / \mathrm{Kg} / \mathrm{min}$, no bolus) infusion. In 4 days we obtained complete resolution of pump dysfunction.

In literature optimal treatment and primary approach for patients with pump thrombosis has not established yet. Support for currently available therapeutic strategies derived from case reports and small case series (single centre experience) [4]. Multiple conflicting reports have been published about the use of fibrinolytics and GpIIb/IIIa, because of the low treatment success, the significant rate of morbidity (hemorrhagic stroke) and death. Moreover Stulak et al. recently observed a significant incidence of pump thrombosis recurrence (57\%) in patients in whom a treatment success was initially obtained [8].

Our philosophy was "to play heavily in attack and then wait in defence". At the beginning, our strategy was to be more aggressive as possible, to save the device, considering the surgical replacement was not feasible. We used all possible treatments to dissolve the clot and to stop the haemolysis.
Obviously the difficulty was to achieve a good balance between necessary anticoagulation and bleeding complications. In fact, while "waiting", the other side of the coin was the huge risk of bleeding linked to the therapy itself, with prolonged hospitalization, the need to change dose of antithrombotic drugs and the subsequent risk of thromboembolic recurrence. In our case after the acute thrombotic event, patient experienced a severe major bleeding from urinary tract and a massive spontaneous hematoma of the psoas muscle. First step was slight reduction in anticoagulant therapy. Then we decided to stop the bleeding with local invasive procedure.

At the end we decided to associate low dose warfarin (INR 2-2.5) with low molecular weight heparin (enoxaparin $4000 \mathrm{UI} / \mathrm{die}$ ) as anticoagulation and Aspirin $100 \mathrm{mg}$ as anti-platelet agent.

There were no more episodes of bleeding or thrombosis for 6 months.

\section{Conclusion}

We report a case of successful medical therapeutic strategy after acute thrombosis of Heart Ware LVAD. Clinical complexity reflects the difficult pharmacologic management of this adverse event, including the ideal agent and dose, the treatment duration and the impact of antithrombotic therapy on subsequent bleeding risk.

However, when patient cannot be a surgical-candidate, drug therapy is the only possible way out.

\section{References}

1. Najjar SS, Slaughter MS, Pagani FD, Starling RC, McGee EC, et al. (2014) An analysis of pump thrombosis events in patients in the HeartWare ADVANCE bridge to transplant and continued access protocol. J Heart Lung Transplant 33: 23-34.

2. Slaughter MS, Rogers JG, Milano CA, Russell SD, Conte JV, et al. (2009) Advanced heart failure treated with continuous flow left ventricular assist device. N Engl J Med 361: 2241e51.

3. Boyle AJ, Russell SD, Teuteberg JJ, Slaughter MS, Moazami N, et al. (2009) Low thromboembolism and pump thrombosis with the Heartmate II left ventricular assist device: analysis of outpatient anticoagulation. J Heart Lung Transplant 28: 881e7.

4. Goldstein DJ, John R, Salerno C, Silvestry S, Moazami N, et al. (2013) Algorithm for the diagnosis and management of suspected pump thrombus. $J$ Heart Lung Transplant 32: 667e70. 
Citation: Perna E, Cipriani MG, Marco DF, Macera F, Milazzo F, et al. (2016) Between Scylla and Charybdis: Difficult Balance between Anticoagulation and Bleeding in the Management of LVAD Thrombosis: A Case Report. Cardiovasc Pharm Open Access 5: 173. doi:10.4172/23296607.1000173

Page 4 of 4

5. Shah P, Mehta VM, Cowger JA, Aaronson KD, Pagani FD (2014) Diagnosis of haemolysis and device thrombosis with lactate dehydrogenase during left ventricular assist device support. J Heart Lung Transplant 33: 102-104.

6. Ravichandran AK, Parker J, Novak E, Joseph SM, Schilling JD, et al. (2014) Haemolysis in left ventricular assist devices: a retrospective analysis of outcomes. J Heart Lung Transplant 33: 44-50.
7. http://www.uab.edu/medicine/intermacs/appendices-4-0

8. Stulak JM, Dunlay SM, Sharma S, Haglund NA, Davis MB, et al. (2015) Treatment of device thrombus in the HeartWare HVAD: Success and outcomes depend significantly on the initial treatment strategy. J Heart Lung Transplant 34: 1535-1541. 